Volume 14, No 1 International Journal of Radiation Research, January 2016

\title{
Impact of contrast agents on dose algorithms of planning systems
}

\author{
M. Izmirli' ${ }^{1}$ T. Çakır ${ }^{*}$, S. Avcu ${ }^{2}$, M. Nart ${ }^{1}$ \\ ${ }^{1}$ Department of Radiation Oncology, School of Medicine, Yuzuncu Yll University, Turkey \\ ${ }^{2}$ Department of Radiology, School of Medicine, Gazi University, Turkey
}

\section{- Original article}

\author{
${ }^{*}$ Corresponding author: \\ Dr. Tahir Çakır, \\ Fax: +904322167519 \\ E-mail: tcakir2003@yahoo.com
}

Revised: May 2015

Accepted: June 2015

Int. J. Radiat. Res., January 2016; 14(1): $25-30$

DOI: 10.18869 /acadpub.ijrr.14.1.25

\section{ABSTRACT}

Background: Use of contrast agents (CAs) during CT simulation for treatment planing system leads to changes in electron density. In this study we aimed to investigate the effect on calculated dose of various concentrations of CAs on treatment planing systems in different dose calculation algorithms. Materials and Methods: Contrast agent $(0.769 \mathrm{mg} / \mathrm{ml}$ lopromid) - water mixtures at concentrations of $0 \%, 1 \%, 2 \%, 5 \%$ and $10 \%$ in total volume of 500 $\mathrm{ml}$ for each were made by using five identical balloons. Calculations were performed by Cobalt-60 and $10 \mathrm{MV}$ linear accelerator devices in CMS XIO treatment planning system. The prescribed dose of $100 \mathrm{cGy}$ was given to the center of balloon that is isocenter of SAD technique at $10 \mathrm{~cm}$ from the surface. The doses at maximum dose depth (dmax) and at $5 \mathrm{~cm}$ were calculated according to the separate algorithms by either making or not making a correction for CA, and the results were recorded. Results: In all algorithms, as contrast ratio increases, the dose values at dmax and $5 \mathrm{~cm}$-depth increase accordingly. When the doses at dmax and $5 \mathrm{~cm}$-depth were compared for Linac and Co-60 in all algorithms, it has been shown that the $d_{\max }$ value of Co60 was higher and the difference was greater in parallel with increasing contrast ratio in comparing with Linac. Conclusion:When required during the planning, the treatment plan should be calculated via providing an electron density correction by contouring the volume retaining CAs along the beam line.

Keywords: Contrast agent, hounsfield unit, electron density, treatment planing system, radiotherapy.

\section{INTRODUCTION}

In CT simulator-assisted CT scans of three-dimensional conformal radiotherapy (3-D CRT) and in more advanced radiotherapy techniques, contrast agents (CAs) are commonly used to more accurately contour the target volumes and off-target normal structures at risk by the help of treatment planning systems. Achieving a good treatment planning is highly dependent on acquiring precise CT images along with using clinical and physical information. Dose calculations are made by employing the treatment planning computer to create a voxel map of electron density of patients in order to use for dose calculations. Tissue containing CAs attenuates the CT X-rays greater than normal, resulting in a higher electron density than the actual density.

Growing body of evidences have reported the effect of CAs on the dose calculation. Ramm et al. reported an overdose of about $7.4 \%$ for the photon beams of $6 \mathrm{MV}$, and of $5.4 \%$ for $25 \mathrm{MV}$ triggered by a bolus diameter of $3 \mathrm{~cm}$ and Hounsfield unit (HU) values of 1400 in a phantom study (1). Many studies using either phantom or patients CT data in 3-D CRT and Intensity modulated radiotherapy (IMRT) treatment planning with various target organs reported the effect of CAs as less than 3\% (2-11). 
In studies, although it is declared that the effect of CAs on dose calculations is ignorable in treatment planning (2-11), a number of authorities suggest the employment of contrast correction, or use of non-contrast CT in radiotherapy planning $(2,6,9,12,13)$.

In the present study, effects of CAs on the alterations of electron density and the effect of these alterations on dose adsorption level in a number of different dose calculation algorithms for each CA concentration were investigated via creating a phantom modelling of $500 \mathrm{cc}$ volume containing CA in various concentrations. Using CMS XIO treatment planning system, the effect of CA on the dose in 3-D treatment plans was determined. By comparing those 3-D treatment plans with the plans acquired after making a contrast correction, it was aimed to minimize the dose increase caused by CA.

\section{MATERIALS AND METHODS}

A mini water phantom with dimensions of $\leftarrow 30 \times 30 \times 30 \mathrm{~cm}$ was built by Plexiglass, and filled with 25 liters of water. Contrast agent (0.769 $\mathrm{mg} / \mathrm{ml}$ Iopromid) - water mixtures at concentrations of $0 \%, 1 \%, 2 \%, 5 \%$ and $10 \%$ in total volume of $500 \mathrm{ml}$ for each were made by using five identical balloons. All balloons were fixed at the same point at the center of the water phantom, and images for CT slices of $5 \mathrm{~mm}$ thickness for each balloon were acquired separately by CT simulator (SIEMENS SOMATOM SENSATION 4). Then, slices were transferred to the CMS XIO 4.34.02 therapy planning system via DICOM. In all sections, 3-D images were obtained by contouring the balloon and phantom edges. Calculations were performed by Theratron 1000E Kobalt-60 teletherapy and SIEMENS Oncor $10 \mathrm{MV}$ linear accelerator devices in the CMS XIO treatment planning system according to the dose algorithm systems of Clarkson, Convolution, Standard Superposition (S. Superposition) and Fast Superposition (F. Superposition).

The doses at maximum dose depth $\left(\mathrm{d}_{\max }\right)$, and at $5 \mathrm{~cm}$ of inhomogeneity contour depth were recorded in a manner that, 100 cGy dose was given to the center of the balloon from the field of $10 \times 10 \mathrm{~cm}^{2}$ generated by a single field SAD $(d=10 \mathrm{~cm})$ technique. The doses calculated separately by either making a correction for CA (the density within the balloon was defined as 1 to the planning system), or making no correction for CA were recorded. $D_{\max }$ dose values and electron densities were recorded as contrast corrected (CC) or non-contrast corrected (NCC) on the axis of the photon beam center belonging to the each algorithm in figure 1. Percent differences of the recorded doses (a-b/b)-100) according to water $(0 \%$ concentration and/or CC) and algorithms were calculated.
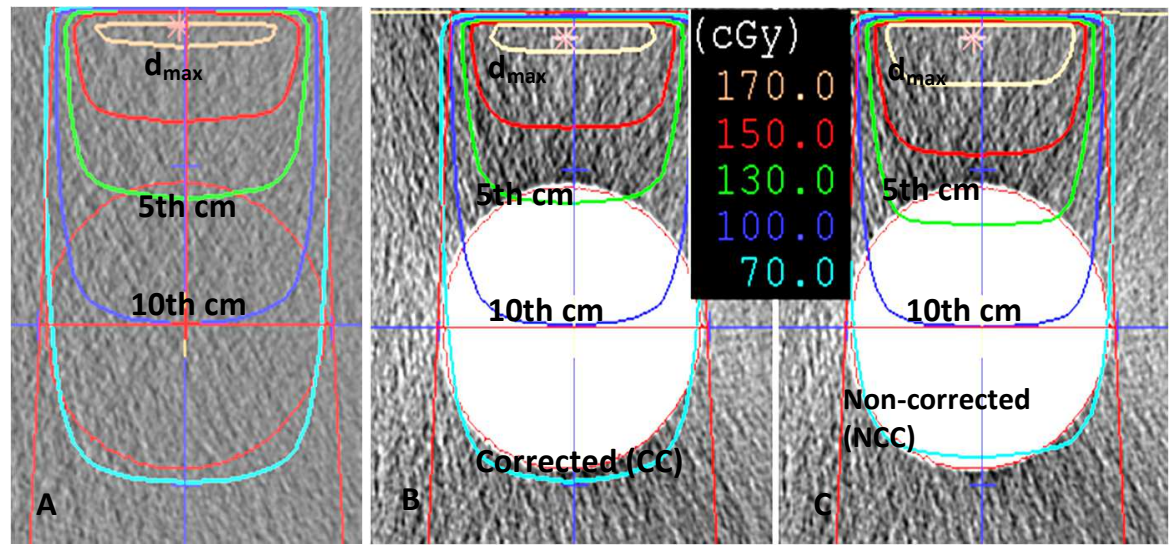

Figure 1. A) Dose distribution with contrast $0 \%, B$ ) Dose distribution with $5 \%$ contrast with contrast-correction and CT section of the beam center, C) Dose distribution with $5 \%$ contrast with no contrast correction and the CT section of the beam center

A: The dose distribution with $0 \%$ contrast, water,

B: Density was adjusted to 1 (water equivalent),

C: Density was kept as is (no adjustment). 


\section{RESULTS}

In all algorithms for dose calculation based on the contrast concentration, electron densities were determined as equal. As it's shown in table 1 , there was no difference between dose values which a contrast correction was made or not in dose calculation algorithms, and in both $\mathrm{d}_{\max }$ and $5 \mathrm{~cm}$-depth in the balloon containing no contrast media. In all algorithms, as contrast ratio increases, the dose values at $d_{\max }$ and $5 \mathrm{~cm}$-depth increase accordingly. When the doses at $\mathrm{d}_{\max }$ and $5 \mathrm{~cm}$-depth were compared for Linac and Co-60 in all algorithms, it's been shown that the $d_{\max }$ value of Co-60 was higher and the difference was greater in parallel with increasing contrast ratio in all algorithms (table 1).

In comparison of the dose calculation algorithms for all contrast ratios at the depth for $\mathrm{d}_{\text {max }}$, the average increase in $D_{\max }$ values between CC and NCC for the Linac $10 \mathrm{MV}$ were as followings for each algorithm: $3.1 \%$ in the Clarkson algorithm, 3\% in the Convolution algorithm, $2.2 \%$ in the S. Superposition algorithm and $2.2 \%$ in the F. Superposition algorithm (table 1, figure 2).

For the Co-60, the average increase in $D_{\max }$ values for each algorithm were $5.8 \%$ in the Clarkson algorithm, 5.5\% in the Convolution algorithm, $3.5 \%$ in the S. Superposition algorithm, and $3.6 \%$ in the F. Superposition algorithm (table 1, figure 3 ).

Table 1. The percent differences of the dose values in calculation algorithms with CC and NCC, and the electron densities.

\begin{tabular}{|c|c|c|c|c|c|c|c|c|c|c|}
\hline \multirow{2}{*}{ Depth } & \multirow[t]{2}{*}{$\%$ Contrast } & \multirow[t]{2}{*}{$\begin{array}{l}\text { Electron } \\
\text { Density }\end{array}$} & \multicolumn{2}{|c|}{$\begin{array}{c}\text { Clarkson } \\
\text { \% Difference (cGy) }\end{array}$} & \multicolumn{2}{|c|}{$\begin{array}{c}\text { Convolution } \\
\text { \% Difference (cGy) }\end{array}$} & \multicolumn{2}{|c|}{$\begin{array}{l}\text { S. Superposition } \\
\text { \% Difference (cGy) }\end{array}$} & \multicolumn{2}{|c|}{$\begin{array}{l}\text { F. Superposition } \\
\text { \% Difference(cGy) }\end{array}$} \\
\hline & & & Linac $10 \mathrm{MV}$ & Co-60 & Linac $10 \mathrm{MV}$ & Co-60 & Linac 10MV & Co-60 & Linac $10 \mathrm{MV}$ & Co-60 \\
\hline \multirow{5}{*}{$d_{\max }$} & 0 & $1.00 * *$ & 0.1 & 0.1 & 0.1 & 0.1 & 0.0 & 0.0 & 0.1 & 0.0 \\
\hline & 1 & 1.08 & 1.1 & 2.2 & 1.0 & 2.0 & 0.8 & 1.3 & 0.8 & 1.2 \\
\hline & 2 & 1.16 & 2.2 & 4.2 & 2.0 & 3.8 & 1.6 & 2.5 & 1.4 & 2.2 \\
\hline & 5 & 1.33 & 4.2 & 7.8 & 4.1 & 7.6 & 3.1 & 4.9 & 3.0 & 4.9 \\
\hline & 10 & 1.57 & 8.1 & 14.5 & 7.9 & 14.2 & 5.6 & 9.1 & 5.8 & 9.1 \\
\hline \multirow{5}{*}{$5 \mathrm{~cm}$} & 0 & $1.00 * *$ & 0.0 & 0.1 & 0.1 & 0.0 & 0.0 & 0.1 & 0.0 & 0.1 \\
\hline & 1 & 1.08 & 1.1 & 2.2 & 1.0 & 2.5 & 0.7 & 1.3 & 0.8 & 2.6 \\
\hline & 2 & 1.16 & 2.2 & 4.2 & 2.0 & 3.8 & 1.5 & 2.8 & 1.4 & 2.3 \\
\hline & 5 & 1.33 & 4.3 & 7.9 & 4.0 & 7.7 & 3.1 & 4.9 & 3.0 & 4.9 \\
\hline & 10 & 1.57 & 8.1 & 14.5 & 7.9 & 14.2 & 5.9 & 9.5 & 5.9 & 9.5 \\
\hline
\end{tabular}

**Contrast correction, electron density in balloons filled with contrast was adjusted to 1 , which is the water equivalent ( $0 \%$ contrast-water). When the dose calculations were made again, it was confirmed that $0 \%$ contrast-water was the same.

Differences among the algorithms at the depth of $d_{\max }$ for Linac (10 MV)

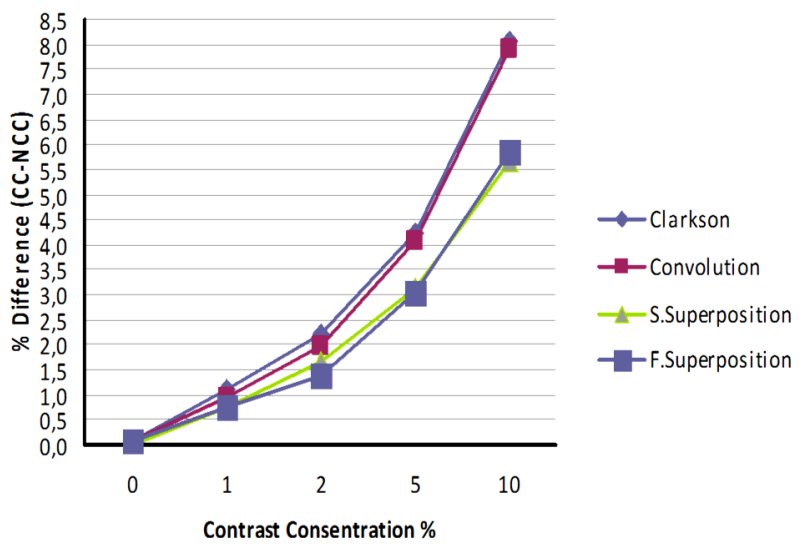

Figure 2. Linac $10 \mathrm{MV}$, comparison of the difference \% among the algorithms for $\mathrm{d}_{\max }$.
Differences among the algorithms at the depth of dmax for Cobalt

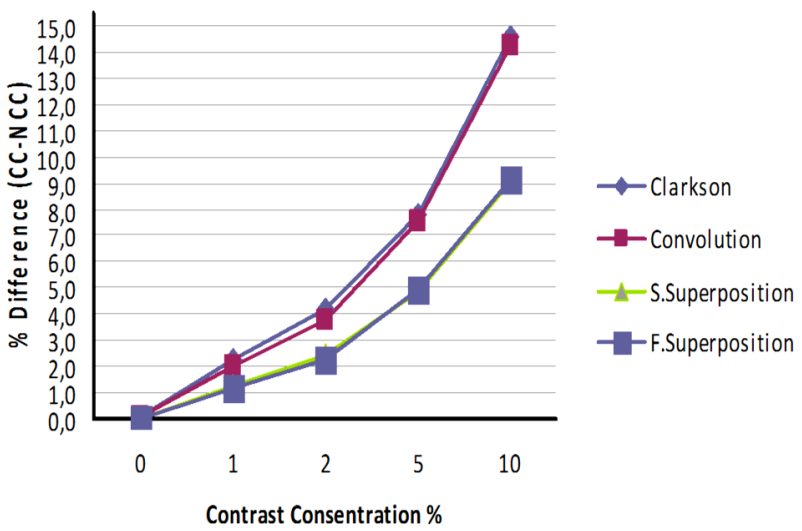

Figure 3. Co- $60 \mathrm{MV}$, comparison of the difference \% among the algorithms for $\mathrm{d}_{\max }$.

Int. J. Radiat. Res., Vol. 14 No. 1, January 2016 
Based on the same comparison between the two groups, the average increases in $\mathrm{D}_{\max }$ values at the $5 \mathrm{~cm}$-depth for the Linac $10 \mathrm{MV}$ were $3.1 \%$ in the Clarkson algorithm, $3 \%$ in the convolution algorithm, and $2.2 \%$ in both the $\mathrm{S}$. Superposition algorithm and the F. Superposition algorithm (table 1, figure 4). For the Co-60, the average increases in $D_{\max }$ values were $5.8 \%$ in the Clarkson algorithm, $5.6 \%$ in the Convolution algorithm, $3.7 \%$ in the $\mathrm{S}$. Superposition algorithm and $3.9 \%$ in the $\mathrm{F}$. Superposition algorithm (table 1, figure 5).

It has been shown that the electron densities determined by treatment planning system increase linearly with the increase of contrast concentration (table 1, figure 6).

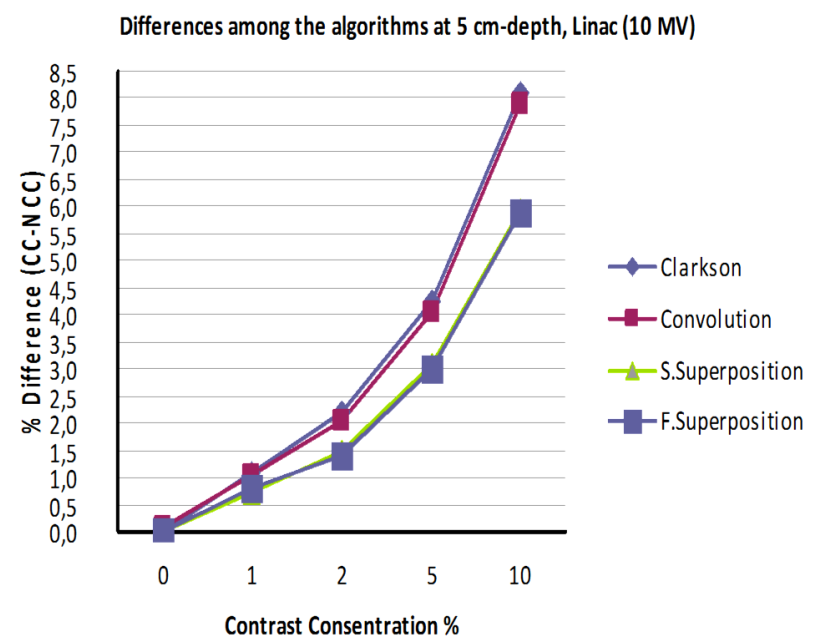

Figure 4. Linac $10 \mathrm{MV}$, comparison of the difference \% among the algorithms for $5 \mathrm{~cm}$.

Differences among the algorithms at $5 \mathrm{~cm}$-depth, Cobalt

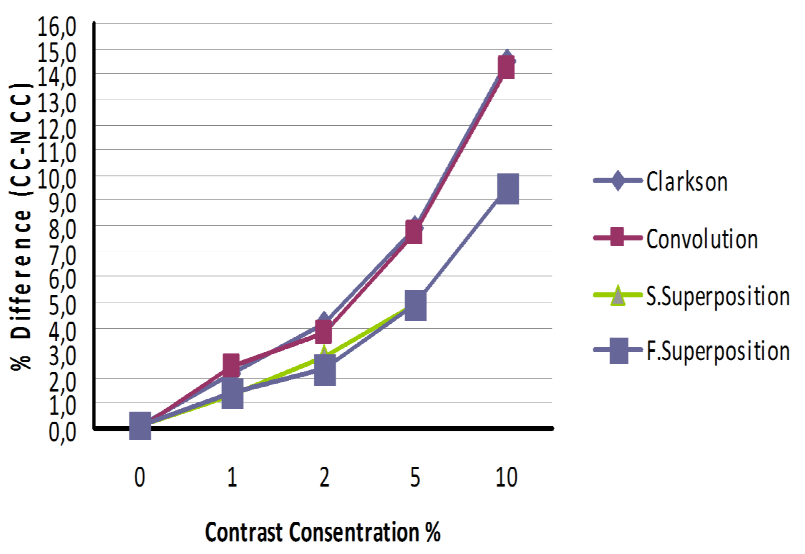

Figure 5. Co-60 MV, comparison of the difference \% among the algorithms for $5 \mathrm{~cm}$-depth.

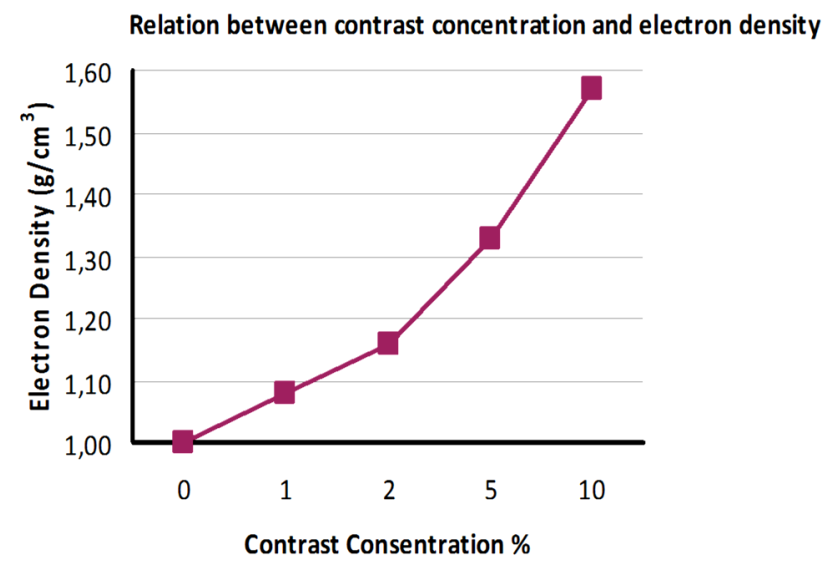

Figure 6. Relationship between contrast concentration and electron density.

\section{DISCUSSION}

Since the use of contrast agents initiates an increment in $\mathrm{HU}$ values, it causes some alterations in electron densities. Alterations in electron densities in slices of CT scans are very important for radiotherapy practice. CAs used in CT simulations may cause an inappropriate evaluation of the dose distribution in patients by affecting organ densities on CT images. Significant relative increases of $\mathrm{HU}$ caused by use of CAs were shown for several targets including liver, kidneys, spleen and thyroid in a range of $22 \%$ to $74 \%$ (14). In present study, the electron density increased with the increasing ratios of CAs, as in the environments with the contrast levels of $0,1,2,5$ and $10 \%$, the electron density measured by all the algorithms were $1.0,1.08,1.16,1.33$ and 1.57 , respectively.

Ramm et al. (1) have observed in their study employing plastic containers in the diameters of 3,6 and $9 \mathrm{~cm}$ containing barium sulfate in varying concentrations that the overdoses of about $7.4 \%$ for the photon beams of $6 \mathrm{MV}$, and of 5.4\% for $25 \mathrm{MV}$ triggered by a bolus diameter of $3 \mathrm{~cm}$ and $1400 \mathrm{HU}$ have occurred. In their study, it has been emphasized that the environment comprising contrast media in smaller than the diameter of $5 \mathrm{~cm}$ and fewer than 500 HU may cause MU increment around 1$3 \%$. In the present study, it was shown that as the electron density of environment increased, the dose differences increased in all algorithms. In the environment with $10 \%$ concentration 
(electron density of 1.57), the dose differences calculated by Clarkson algorithm for $10 \mathrm{MV}$ and Co60 were determined as $8.1 \%$ and $14.5 \%$, respectively. In the conditions with the concentrations of $1 \%$ and $2 \%$ (electron density of 1.08 and 1.16, respectively), the dose differences acquired via Clarkson algorithm for $10 \mathrm{MV}$ and Co60 were found as $1.1-2.2 \%$ and $2.2-$ $4.2 \%$, respectively ${ }^{(1)}$.

A number of studies carried out on phantom have determined the effect of the dose of CAs varying between $2-7 \%(12,15,16)$. Lees et al. (15) have investigated dose alterations calculated at various gantry angles by the XIO (version 4.1.1) super position algorithm on the contrast-enhanced or noncontrast-enhanced CT sections belonging to the three patients for each on a phantom, and have documented fewer than $2 \%$ increment in calculated doses with the contrast use. In that study, it has been suggested that the use of contrast may have an effect of a minimal dose increment on the treatment planning system.

In many studies, it has been foreseen that the contrast use in head and neck treatment plans could cause very small, negligible dose differences on dose distribution in the IMRT treatment plan (2-5). In a study conducted by Letourneau et al. (2), a comparison in the doses received by the target organs and the organs at risk was made, and the differences between the two dose calculations (before and after the density alteration) were evaluated by replacing the increased densities of blood vessels with contrast to water equivalent $\left(1.00 \mathrm{~g} / \mathrm{cm}^{3} \mathrm{HU}\right.$ of 0 ) on acquired contrast-CT images. In this study, a clinically relevant 'no contrast' model has been achieved, and the change in planning target volume (PTV) coverage was kept minimum between the plans with and without contrast. The volume of the PTVs covered by the $93 \%$ and $100 \%$ isodoses changed on average by $0.57 \%$. The minimum dose to PTVs varied by a maximum of $0.17 \mathrm{~Gy}$. The maximum point dose to critical organs changed by a maximum of 0.12 Gy (brainstem). Non-physiologic extremes of density within blood vessels also resulted in minimal changes in tumor or normal tissue dosimetry. The use of IV contrast at time of CT-simulation does not significantly affect dose calculation in head and neck IMRT plans. In our study, based on the contrast concentrations in the developed phantom for dose calculation in all algorithms, the electron densities were determined as equal. No difference was recorded between the doses in which either a contrast correction was made or not in dose calculation algorithms, and in both $\mathrm{d}_{\max }$ and $5 \mathrm{~cm}$-depth in the balloon containing no contrast media. In all algorithms, the dose values at $d_{\max }$ and $5 \mathrm{~cm}$-depth increased in parallel with increasing contrast ratios. When the doses at $\mathrm{d}_{\max }$ and $5 \mathrm{~cm}$-depth were compared to Linac and Co-60 in all algorithms, the $d_{\max }$ value of Co-60 was higher and the difference was greater accordingly to the increasing contrast ratio in all algorithms (table 1).

In treatment plans made by 3-DCRT and IMRT, an effect of CAs was investigated in lung cancers, and its effect on dose was determined negligible (3\% at maximum) ${ }^{(6-9)}$. In a study conducted by Fayda et al. (6) on patients with lung cancer, the dose changes of CAs for simulation purposes calculated by various algorithms in two different treatment planning systems were evaluated. It was shown that the mean MU change was $+1.3 \%$ $(-1 \%$ to $+3.2 \%)$. Based on this outcome, it was suggested that 3D planning of the lung would better be performed by combinatory use of non-contrast CT and contrast-CT in treatment planning and creating volumes, respectively.

In another study, Shibamoto et al. (10) investigated MU changes caused by contrast use in CT planning for brain, neck, upper abdomen and pelvic area. In all areas except upper abdomen, it was shown that contrats retention did not cause any significant MU change. In upper abdomen, though, significant dose increments up to $2 \%$ were reported, especially with the contrast retention in the right lobe of liver.

In their patient-oriented study conducted with Cyberknife, Kim et al. (17) have found a difference around $2 \%$ in dose distribution with contrast use in Cyberknife treatment plan, but it was also emphasized that the differences increased up to $7.8 \%$ based on the target position. Besides the effect of contrast use, a greater difference as high as $20 \%$ could be seen owing to the calculation algorithms.

According to these results, although it is declared that the effect of CAs on dose

Int. J. Radiat. Res., Vol. 14 No. 1, January 2016 
calculations is ignorable in treatment planning (2-11), a number of authorities suggest the employment of contrast correction, or use of non-contrast CT in radiotherapy planning $(2,6,9,12,13)$. Consequently, under the shed of the current findings, it is considered that 3-D radiotherapy planning can be performed using contrast-CT slices, and dose alterations occurred in target volumes are negligible. On the other hand, it should be remembered that, depending on causing an increase in electron density, an addition of contrast agent may cause dose increments in the organs at risk for various area corrections and algorithms in a personal manner. When required (especially situations in which electron density exceeds), dose should be calculated again after making a correction of electron density via contouring volume retaining contrast on the beam way in a planning system.

\section{Conflicts of interest: none to declare.}

\section{REFERENCES}

1. Ramm U, Damrau M, Mose S, Manegold KH, Rahl CG, Böttcher HD (2001) Influence of CT contrast agents on dose calculations in a 3D treatment planning system. Phys Med Biol, 46(10): 2631-2635.

2. Létourneau D, Finlay M, O'Sullivan B, et al. (2008)Lack of influence of intravenous contrast on head and neck IMRT dose distributions. Acta Oncol, 47: 90-4.

3. Lee FK, Chan CC, Law CK (2009) Influence of CT contrast agent on dose calculation of intensity modulated radiation therapy plan for nasopharyngeal carcinoma. J Med Imaging Radiat Oncol, 53(1): 114-118.

4. Choi Y, Kim JK, Lee HS, Hur WJ, Hong YS, Park S, Ahn K, Cho $H(2006)$ Influence of intravenous contrast agent on dose calculations of intensity modulated radiation therapy plans for head and neck cancer. Radiother Oncol, 81(2): 158-162.

5. Liauw SL, Amdur RJ, Mendenhall WM, et al. (2005)The effect of intravenous contrast on intensity-modulated radiation therapy dose calculations for head and neck cancer. Am J Clin Oncol, 28: 456-9.

6. Fayda M, Yıldırım A, Tarpıcı N, Aksu G, Çakır A (2008) The effect of intravenous contrast agent on lung cancer treatment planning with different systems. Turkish Journal of Oncology, 23(3): 126-131.

7. Xiao J, Zhang $\mathrm{H}$, Gong $\mathrm{Y}$, Fu Y, Tang B, Wang S, Jiang $\mathrm{Q}$, Li P (2010) Feasibility of using intravenous contrast-enhanced computed tomography (CT) scansin lung cancer treatment planning. Radiother Oncol, 96: 73-77.

8. Shi W, Liu C, Lu B, Yeung A, Newlin HE, Amdur RJ, Olivier KR (2010) The effect of intravenous contrast on photon radiation therapy dose calculations for lung cancer. Am J Clin Oncol, 33: 153-156.

9. Burridge NA, Rowbottom CG, Burt PA (2006) Effect of contrast enhanced CT scans on heterogeneity corrected dose computations in the lung. J Appl Clin Med Phys, 7: 112.

10. Shibamoto $Y$, Naruse A, Fukuma H, Ayakawa S, Sugie $C$, Tomita N (2007) Influence of contrast materials on dose calculation in radiotherapy planning using computed tomography for tumors at various anatomical regions: a prospective study. Radiother Oncol, 84: 52-55.

11. Weber DC, Rouzaud M, Miralbell R (2001) Bladder opacification does not significantly influence dose distribution in conformal radiotherapy of prostate cancer. Radiother Oncol, 59: 95-97.

12. Rankine AW, Lanzon PJ, Spry NA (2008) Effect of contrast media on megavoltage photon beam dosimetry. Med Dosim, 33: 169-174.

13. Zabel-du Bois A, Ackermann B, Hauswald H, Schramm O, Sroka-Perez G, Huber P, Debus J, Milker-Zabel S (2009) Influence of intravenous contrast agent on dose calculation in 3-D treatment planning for radiosurgery of cerebral arteriovenous malformations. Strahlenther Onkol, 185: 318 $-324$.

14. Amato $E$, Lizio $D$, Settineri $N$, et al. (2010) A method to evaluate the dose increase in CT with iodinated contrast medium. Med Phys, 37: 4249-56.

15. Lees J, Holloway L, Fuller M, Forstner D (2005) Effect of intravenous contrast on treatment planning system dose calculations in the lung. Australas Phys Eng Sci Med, 28(3): 190-195.

16. Robar JL, Riccio SA, Martin MA (2002) Tumour dose enhancement using modified megavoltage photon beams and contrast media. Phys Med Biol, 47: 2433-2449.

17. Kim HJ, Chang AR, Park YK, Ye SJ (2013) Dosimetric effect of CT contrast agent in Cyber Knife treatment plans. Radiat Oncol, 8(1): 24. 